\title{
Further development of spinal cord retreatment dose estimation: including radiotherapy with protons and light ions
}

\author{
Joshua W. Moore, Thomas E. Woolley, John W. Hopewell \& Bleddyn Jones
}

To cite this article: Joshua W. Moore, Thomas E. Woolley, John W. Hopewell \& Bleddyn Jones (2021): Further development of spinal cord retreatment dose estimation: including radiotherapy with protons and light ions, International Journal of Radiation Biology, DOI: 10.1080/09553002.2021.1981554

To link to this article: https://doi.org/10.1080/09553002.2021.1981554

View supplementary material $ひ$

Accepted author version posted online: 15

Sep 2021.

Submit your article to this journal 지

Q View related articles 두

View Crossmark data $₫$ 


\section{Further development of spinal cord retreatment dose estimation: including radiotherapy with protons and light ions.}

Joshua W. Moore ${ }^{\mathrm{a}}$, Thomas E. Woolley ${ }^{\mathrm{a}}$, John W. Hopewell ${ }^{\mathrm{b}}$ and Bleddyn Jones ${ }^{\mathrm{b}, \mathrm{c}^{*}}$. ${ }^{a}$ Cardiff School of Mathematics, Cardiff University, Senghennydd Road, Cardiff, UK. ${ }^{\mathrm{b}}$ Green Templeton College, University of Oxford, Oxford, UK.

${ }^{c}$ Gray Laboratory, Department of Oncology, University of Oxford, Old Road Research Campus, Oxford, UK

*Correspondence to Prof Bleddyn Jones: Gray Laboratory, Department of Oncology, University of Oxford, Old Road Research Campus Research Building, Roosevelt Drive, Oxford OX3 7DQ, UK

E-mail: Bleddyn.Jones@ oncology.ox.ac.uk

Keywords: radiobiology; retreatment; radiotherapy; protons; ions.

Running title: Spinal cord retreatment including protons and ions 


\begin{abstract}
Purpose: A graphical user interface (GUI) was developed to aid in the assessment of changes in the radiation tolerance of spinal cord/similar central nervous system tissues with time between two individual treatment courses.
\end{abstract}

Methods: The GUI allows any combination of photons, protons (or ions) to be used as the initial, or retreatment, radiotherapy courses. Allowances for clinical circumstances, of reduced tolerance, can also be made. The radiobiological model was published previously and has been incorporated with additional checks and safety features, to be as safe to use as possible. The proton option includes use of a fixed RBE of 1.1 (set as the default), or a variable RBE, the latter depending on the proton linear energy transfer (LET) for organs at risk. This second LETbased approach can also be used for ions, by changing the LET parameters.

Results: GUI screenshots are used to show the input and output parameters for different clinical situations used in worked examples. The results from the GUI are in agreement with manual calculations, but the results are now rapidly available without tedious and error-prone manual computations. The software outputs provide a maximum dose limit boundary, which should not be exceeded. Clinicians may also choose to further lower the number of treatment fractions, whilst using the same dose per fraction (or conversely a lower dose per fraction but with the same number of fractions) in order to achieve the intended clinical benefit as safely as possible.

Conclusions: The new GUI will allow scientific-based estimations of time related radiation tolerance changes in the spinal cord and similar central nervous tissues (optic chiasm, brainstem), which can be used to guide the choice of retreatment dose fractionation schedules, with either photons, protons or ions.

\title{
NOTE TO REVIEWERS
}

A standalone Graphic User Interface is provided with this manuscript to aid calculation based on any combination of photon/proton (ion) treatment and retreatment. This can be downloaded using the following link https://www.dropbox.com/sh/bsdt57c07n2smw4/AABvryRbu-4815zSnxYOIHaOa?dl=0 where additional instruction can also be obtained. 


\section{Introduction}

The decision to retreat an already irradiated volume of tissue is amongst the most difficult in clinical radiotherapy. Furthermore, the choice of dose-fraction schedule is especially demanding, especially in neural tissue where late reacting tissue damage can be life changing or even fatal. Few guidelines have emerged from many published clinical studies, which typically neither record the time interval between treatments nor specify the biological effective doses (BED) used. For examples, readers should note the entire contents of a special edition of the journal 'Clinical Oncology' which followed the editorial by Muirhead and Jones (2018).

Following personal experience of the retreatment of brain and spinal tumours, following the emergence of experimental radiobiological data which showed a time dependent recovery of neural radio-tolerance, mathematical models were developed in the UK to further simulate this process (Jones and Grant 2014). A series of further publications have refined the development of a time-interval dependent model for estimating changes in spinal cord retreatment radiation tolerance (Jones and Hopewell 2014; Woolley et al. 2018; Jones and Hopewell 2019) including the use of a preliminary Graphical User Interface (GUI) for photon-based retreatment calculations (Jones and Hopewell 2019; Woolley et al. 2018). Such models have been used to guide clinicians in Oxford and at some other UK Centres since 2014 and thus far the present authors are not aware of any adverse sequelae, although no formal audit has been performed. The model was always applied cautiously (or in a conservative manner) with respect to dose-fractionation and normal tissue tolerance, as described below.

The model developed incorporated all known published radiobiological experimental data sets (in laboratory rats and primates) which investigated the influence of time on the recovery of neural radio-tolerance. These data sets are listed in Jones and Hopewell 
(2019) and Woolley et al. (2018) and the human dose response curve for radiation myelopathy (from the late Kian Ang, MD Anderson Cancer Center) was used as the baseline for human studies, with the known time lag of 70 days before recovery of radio-tolerance commences (Woolley et al. 2018). Use of the latter prevents overdosage with shorter intervals between two separate treatment courses. From the human data set, a maximum permitted tolerance dose of $50 \mathrm{~Gy}$ in 25 fractions is used for otherwise fit individuals with no adverse medical or surgical medical histories (these are considered further below). This tolerance level is a standard assumption within radiotherapy and has been recognised for several decades (Van der Kogel 1989; Schultheiss et al. 1995) and is supported by the human dose response data mentioned above. However, many radiation oncologists elect to lower this level according to the perceived risk. The BED concept is used to compare fractionation schedules (Jones et al. 2001). For example, a tolerance dose of $50 \mathrm{~Gy}$ in 2 Gy fractions has a BED of 100 $\mathrm{Gy}_{2}$, where the subscript denotes the $\alpha / \beta$ ratio of $2 \mathrm{~Gy}$. The BED concept description and its applications are described elsewhere (Jones et al. 2001).

Radiation oncologists may choose to use an $8 \%$ reduction to $92 \mathrm{~Gy}_{2}$ by prescribing a more conservative dose of 46 Gy in 23 fractions; or even more conservatively, the use of $45 \mathrm{~Gy}$ in 25 fractions which results in a BED of $85.5 \mathrm{~Gy}_{2}$, a $14.5 \%$ reduction.

More detailed references and explanations are given in the above series of publications and users of the model should familiarise themselves with these. The model contains many important features selected to protect a patient from over-dosage caused by breach of the allowed tolerance level. It can compensate for important clinical factors, which are known to influence radiation tolerance such as the extremes of age, previous surgery, extensive chemotherapy exposures and significant concomitant medical disorders in the medical history. These factors are well known to radiation oncologists, 
and frequently lead to the modification of treatment prescription when there is serious concern. Within the model, these can be included by use of the so-called 'conservative factor' which allows up to a $20 \%$ reduction in the biological effective dose (BED) associated with the accepted tolerance level according to the perception of risk judged by the treating radiation oncologist. For example, significant surgery in the irradiated region might carry a $10 \%$ reduction as might also high dose chemotherapy exposures. Extremes of age might warrant a 5-10\% reduction, as might the presence of diabetes mellitus, other vasculopathy disorders or chronic renal failure where delayed healing can be expected. Application of the model is consequently dependent on a thorough clinical assessment and allocation of the 'clinical' tolerance level. Radiation oncologists routinely make such allocations and the influence of such clinical factors, in terms of BED, have been reported elsewhere (Jones et al. 2006). Radiobiology textbooks, such as Hall and Gaccia (2019), advise caution in irradiating the spinal cord when given simultaneously or sequentially with cytotoxic drugs such as methotrexate, cis-platinum, vinblastine and cytosine arabinoside, which have their own neurotoxic properties. Further reductions in tolerance, at the extreme of age, have been noted in experimental animal studies (Ruifrok et al. 1994), and this is consistent with experience in humans.

Although the use of proton therapy was included in the most recent publication (Jones and Hopewell 2019), with allowances for linear energy transfer (LET) and relative biological effectiveness (RBE), the retreatment dose fractionation estimations required further long manual calculations to be carried out independently of the early version of the GUI available at that time (Woolley et al. 2018). The new GUI now provides these solutions, thus obviating the need for any such calculations.

The present paper describes a new version of the GUI, which allows retreatment dose fractionation estimations for the spinal cord (and regions of the brain with similar 
radiation tolerance) for any combination of initial treatment and retreatment using photons, or protons, including the option of two photon courses, or two proton courses. Ion beams can be substituted for protons if these are available options. In both cases $\mathrm{RBE}$ corrections are available within the dose-fraction estimations in order to make the retreatment dose estimations more conservative. These are described further below.

Further improvements in the software systems have also been achieved, as given below.

\section{Methods}

\section{Further Development of the BED calculator}

The current GUI has been developed in Java, using the JavaFX library along with Scene Builder to create the actual User Interface (UI). This means that the GUI can be used on any operating system and the file size is extremely low (approximately 100kb), which enables the file to be easily downloaded for clinical use, resolving previous accessibility issues since the original BED calculator was dependent on licensed mathematical software, Matlab (Woolley et al. 2018). The new version was designed to have a similar aesthetic to the original version, created in Matlab (Woolley et al. 2018), to allow for a seamless transition to the new calculator. There are small changes regarding notation, therefore a notation popup was added to reduce potential user ambiguity (Appendix 1)

The time-dependent model for photon radiotherapy, developed previously as described in Woolley et al. 2018, was used, allowing the same numerical techniques to be used to solve for appropriate values. To include the use of proton and ion radiotherapy in the GUI, the proton (or ion) doses were converted into photon equivalent doses by using either the conventional standard (fixed) RBE of 1.1 (for protons), or a variable LET-dependent RBE as the dose conversion factor, as developed 
elsewhere (Jones and Hopewell 2019; Jones 2015a; Jones 2015b; Jones 2017). This allows for higher, normal tissue protective RBE values, than the conventional standard value of 1.1, in the mid spread-out Bragg peaks and for higher LET values. This is specially the case at low doses per fraction, when with a $\alpha / \beta$ ratio of $2 \mathrm{~Gy}$, the resulting iso-effective doses will be lower. This option thus offers further conservatism compared with the standard international assumption of a proton RBE of 1.1, at all dose levels. This assumption and the way in which it was derived has been criticised (Jones 2016) and there now appears to be greater consensus that a better alternative is necessary (Paganetti et al. 2019). These two RBE approaches can be used within the GUI and can be applied for each combination of photon and proton (or ion) retreatments depending on the view of the user. Some users may wish to apply a compromise between these two approaches (i.e. adopt a dose and fraction number which is intermediate between the predictions of each system). For further information on the retreatment dose estimation models integrated into the present software, see Appendix 2.

The GUI is partitioned into four sections containing each of the potential combinations of treatment sequences mentioned above. Each window runs independently of the others and, consequently, the most recent calculation is stored in its specific window, this enables the user to compare outputs from each treatment combination with minimal effort.

For proton (ion) retreatments, the output of the GUI was changed to provide the number of retreatment fractions for a given proton (ion) retreatment dose per fraction, whereas for photon retreatments the retreatment dose per fraction is the output for a preselected number of retreatment fractions. This change is necessary because the retreatment dose per fraction $\left(d_{r e t}\right)$ and if used the operative LET must be specified by the user in order to determine the appropriate RBE. Therefore, the only unknown parameter required to estimate the retreatment $\operatorname{BED}\left(B E D_{\text {ret }}\right)$ is the number of 
retreatment fractions, $n_{\text {ret }}$. The number of retreatment dose fractions, in clinical practice, can only be a positive integer, but the method used allows for $n_{\text {ret }}$ to be a noninteger. Thus, a 'dose adjustment' feature was implemented to modify $d_{r e t}$ so that $n_{r e t}$ rounds to the nearest integer. Using the rounded $n_{r e t}$ a standard mathematical bisection method was used to solve for small adjustments to $d_{\text {ret }}$ required for the given $B E D_{\text {ret }}$ value (Chapra 2012). Further 'catches' were developed to inform the user if the suggested dose per fraction changes the RBE value, namely, the RBE output will flash to gain the attention of the user. It is advised that the user first arrives near (within \pm 0.5 ) the desired $n_{\text {ret }}$ value, then turn on the dose adjustment feature to fine tune the retreatment dose for the integer $n_{r e t}$. It should be noted that the displayed retreatment dose per fraction is rounded to 2 decimal places in order to be consistent with the photon retreatment windows.

As in the original GUI, various safety procedures have been incorporated into the software. Every input box prevents the input of inappropriate values (Woolley et al. 2018). If such an inappropriate value was used in any input box, activation of calculation will change the value back to that of the input default. In addition to this, warnings to indicate any initial over-dosage or risks of high probabilities of myelopathy have been introduced. Accompanying these features, by utilising the model developed previously (Woolley et al. 2018), the conservative factor box shifts are also present for proton (or ion) radiotherapy. The most critical of the patient-related inputs (years before retreatment, the conservative factor and retreatment dose per fraction or number of fractions) have been highlighted using borders. These inputs are left empty on launching the application and it requires the user to manually set these important parameters. If the user was to leave one of these empty then on starting a calculation, a warning would appear, and the critical empty input box would flash. 


\section{Using the GUI}

The first requirement of the user is to choose the appropriate combination of initial and retreatment options. Each possible combination of initial and retreatment options correspond to one of the tab titles (see SUPPLEMENTARY MATERIAL 1 and 2 for information files and to view the GUI structure and layout). A table of parameters is available within the software and is now included in Appendix 2.

Once the appropriate treatment sequence has been selected it is necessary to allocate a percentage risk of myelopathy acceptable on retreatment. The previous default value was set at $1 \%$, but most users preferred $0.1 \%$ (the revised default), or a value of $0.1185 \%$, which is the risk associated with the standard assumption that tolerance is associated with a BED value of $100 \mathrm{~Gy}_{2}$ (25 dose fractions of 2 Gy per fraction, total dose $50 \mathrm{~Gy}$ ). The dose-fractionation details of the initial treatment are then inserted. The time interval between the initial treatment and the proposed retreatment needs to be entered, along with any clinical requirement to change the conservative factor (Woolley et al. 2018). The proposed number dose fractions for retreatment with photons is then entered, or for all treatments using protons it is necessary to accept one of the two following methods of working:

(1) To accept a fixed RBE of 1.1 (the currently accepted standard practice in many centres). This is currently set as the default by the tick in the appropriate box, or can be unticked to:

(2) Allow the provision of an appropriate LET value for central nervous tissue, which is converted to an operative RBE value. This also changes the tolerance doses using the equations published previously (Jones and Hopewell 2019, Jones 2015a; Jones 2017). This option will provide further protection against 
any inadvertent over-dosage at low doses per fraction, if RBE values exceed 1.1 in the CNS (Lu hr et al. 2017; Saager et al. 2018).

For ion beam based treatments, it is always necessary to allocate an operative $L E T_{u}$ value (the LET at which RBE is maximal over the entire LET range for any given dose). Recommended values of $L E T_{u}$, published elsewhere (Jones and Hill, 2019), are included in the supplementary material (SUPPLEMENTARY MATERIAL 3). The simple energy efficiency model is used, as in the case of protons. Alternative, but more complex predictive models, which would require the input of additional parameters are available (Elsasser et al. 2008; Friedrich et al. 2013; Cheng et al. 2017). There appears to be no consensus as to which model is best prior to large comparative experimental programmes being undertaken.

The choice of an appropriate reference radiation LET (the $L E T_{c}$ value) is also important and should be based on a comparison with megavoltage photons used in the clinic (range $0.2-0.6 \mathrm{keV} / \mu \mathrm{m}$ ). The default value is set at $0.22 \mathrm{keV} / \mu \mathrm{m}$. It should be appreciated that for ortho-voltage x-rays the LET values can significantly exceed $1 \mathrm{keV} / \mu \mathrm{m}$, especially if poorly filtered, resulting in proton RBE values less than 1.0 in the mid spread-out Bragg peak (SOBP) (Jones 2016). The SOBP regions normally have LET values of $1-2 \mathrm{keV} / \mu \mathrm{m}$, but significantly higher values are found towards the end of the SOBP. Values between $2-10 \mathrm{keV} / \mu \mathrm{m}$ may be found in normal tissues exposed to lower doses outside the Bragg peak region with scanned proton beams (Grassberger et al. 2012). These regions need careful clinical consideration, as suggested previously (Jones and Hopewell 2019), since higher LET values increase the RBE.

On completing the input values, the 'Calculate' button will become active. Once pressed, the GUI will compute the required solution and will provide one of two 
outputs, depending on whether retreatment is with photons or protons. In the case of photon retreatments the dose per fraction is displayed, for the requested number of dose fractions. Alternatively, in the case of proton (or ion) retreatment the number of dose fractions is displayed, as a non-integer is displayed if 'turn off dose adjustment' is checked. Unchecking the 'turn off dose adjustment' will allow the GUI to adjust the dose per fraction, providing a modified dose per fraction size that results in the required full integer fraction number.

The number of fractions proposed for retreatment can be changed and iterations of different dose estimations can be obtained for different conditions, e.g. for alternative treatment plans with different LET values.

When carrying out repeated estimations, it is important to check that the values of the variable parameters remain appropriate. It is recommended that two people with experience in the use of the GUI should check on the parameter entries and the final estimation for clinical use must be interpreted according to the clinical circumstances.

\section{Results}

In order to help new users, window displays are given for various worked examples, largely based on examples used in a previous publication (Jones and Hopewell 2019). These are not actual clinical cases but are designed to provide experience in use of the GUI and its various features. As a training exercise, users are encouraged to replicate the further examples given in the same publication.

Various treatment combinations are used in Examples 1-4 (Figures 1-4), and an additional example of a carbon ion retreatment is given in Example 5 (Figure 5). In 
Example 5 it is necessary to open the fourth (proton and proton option) and then adjust the LET settings.

\section{Example 1: Initial photon treatment followed by photon retreatment}

This is the same example as used with the previously version of the GUI (Woolley et al. 2018), where the patient received an initial spinal cord dose of 46.5 Gy in 30 fractions. Retreatment for recurrence was required 18 months later. Retreatment was proposed using 20 fractions, with a conservative factor of $10 \%$, due to two previous courses of high dose chemotherapy. Figure 1 shows where these inputs have been added as well as the calculated initial dose per fraction $(1.55 \mathrm{~Gy})$ and the retreatment dose per fraction of $1.9 \mathrm{~Gy}$, all based on a retreatment myelopathy risk of $0.1 \%$ (the BED is $88.36 \mathrm{~Gy} 2$ after taking into account the additional risk due to chemotherapy). For further explanation of the parameters $s_{0}, s_{1}$ and $\underline{B E D}$, readers should consult the previous publication (Woolley et al. 2018).

\section{Example 2: Initial photon treatment followed by proton retreatment}

The first example of this category uses a known LET allocation while the second example uses a fixed RBE of 1.1 for the same clinical situation. After an initial photon dose of $47.5 \mathrm{~Gy}$ in 30 fractions to the spinal cord, a proton retreatment is proposed 18 months later. There were no adverse clinical features in the medical history, which indicates that full radio-tolerance can be expected, so the conservative factor used was set at $0 \%$. In the first example (Figure $2 \mathrm{a}$ ) the proton LET was $1.5 \mathrm{keV} / \mu \mathrm{m}$ and the required dose per fraction was entered as 1.6 Gy. The initial output showed a noninteger number of fractions (23.93), so the calculation was repeated with a proton dose of $1.5968 \mathrm{~Gy}$ to be given for 24 fractions. It should be noted that in the publication (Jones and Hopewell 2019), a different approach was used, the number of fractions of 
1.6 Gy were rounded down to 23 as a 'fall safe' mechanism (i.e. a deliberate choice is made to reduce the potential for toxicity by rounding down on the number of fractions rather than rounding up). The same fall safe principle applies to dose per fraction which may also be further reduced beyond that given by the model on the advice of the treating radiation oncologist.

In the second example for this treatment combination, the 'fix retreatment RBE of 1.1' was left as the default. The output result for a proton retreatment dose per fraction of $1.6525 \mathrm{~Gy}$ is 24 fractions. This approach results in a higher total dose being delivered in 24 fractions. The difference total dose delivered was $1.32 \mathrm{~Gy}$, if very accurate doses per fraction were used. Alternatively, the difference in total dose delivered was $1.25 \mathrm{~Gy}$, if the clinically used doses per fraction were rounded to 1.65 and 1.6 Gy, respectively. The smaller dose per fraction of $1.5968 \mathrm{~Gy}(1.6 \mathrm{~Gy})$ resulted from using an operative RBE of 1.14 as opposed to the fixed value of 1.1. For higher LET values the worked examples in a previous publication (Jones and Hopewell 2019) should be followed.

\section{Example 3: Initial proton treatment followed by photon retreatment}

This example duplicates worked example 6 from previous publication (Jones and Hopewell 2019) for an initial proton treatment of 39 Gy in 30 fractions to the optic chiasm, with an operative LET of $1.5 \mathrm{keV} / \mu \mathrm{m}$. Retreatment was required two years after the initial treatment. There is no adverse medical history (so the conservative factor in the GUI is set at $0 \%$ ). The RBE is displayed as 1.15 and for a required 30 photon retreatment fractions, the dose per fraction output is 1.66 Gy (Figure 3).

\section{Example 4: Initial proton treatment followed by proton retreatment}


Here, it is assumed that the first treatment has an LET of $1.3 \mathrm{keV} / \mu \mathrm{m}$ and the retreatment $1.8 \mathrm{keV} / \mu \mathrm{m}$. The conservative factor is set by the clinician at $10 \%$ (due to the medical history) and retreatment is given 2.5 years after the initial treatment of $38.5 \mathrm{~Gy}$ in 30 fractions to the spinal cord. The output shows 22 fractions of $1.6747 \mathrm{~Gy}$. In this case, the initial dose per fraction for retreatment used was $1.6 \mathrm{~Gy}$ but in order to obtain an integer number of fractions the dose per fraction was increased slightly.

\section{Example 5: Initial proton treatment followed by carbon ion retreatment}

In this example two carbon ion treatments were required 2.5 years apart with a clinician demand for a conservative factor of $10 \%$. The maximum bio-efficiency LET was set at $150 \mathrm{keV} / \mu \mathrm{m}$ for both the initial and retreatment doses. The operative LET was assumed to be 50 and $60 \mathrm{keV} / \mu \mathrm{m}$ for the initial treatment and retreatment, respectively. The initial treatment delivered to a total dose of $10 \mathrm{~Gy}$, in 15 fractions, to the spinal cord. Retreatment was requested using $1.5 \mathrm{~Gy}$ dose-fractions. The calculation provided a dose per fraction of 1.5007 Gy consistent with 11 fractions, as seen in Figure 5.

\section{Discussion}

The new GUI described above provides a practical platform for obtaining retreatment dose-fractionation estimations for the spinal cord and other areas of the central nervous system with similar radiation tolerance, such as the brainstem and optic chiasm regions. It removes the need to do any manual calculations, which have many separate steps and pitfalls, especially when the added difficulties presented by increasing LET and RBE are involved.

The system aims to provide a safe upper limit to the retreatment dose per fraction for a given number of fractions, but continues to need considerable clinical 
input to define the acceptable risk level for a given retreatment situation, and to determine the degree of conservative factor allocation (a BED reduction allocation according to clinical circumstances, as discussed previously (Jones and Hopewell 2019; Woolley et al. 2018)). Further, clinical judgement may be required to operate in a 'fall safe' way, for example by using a lower number of retreatment fractions or a reduced dose per fraction than suggested by the model, if there is any doubt. The upper limit provided by the model is more appropriate for radical retreatment situations. For palliative situations there is considerable leeway for reductions in dose per fraction or fraction number in order to deliver a schedule that has a high probability of achieving the intended effect, such as relief of pain or bleeding, which require a lower BED. Such situations are often delivered using fraction sizes greater than 2 Gy and even single treatment sessions in some countries.

The system presented has used only one LET-RBE model, but this is based on results from extensive biological data sets, with wide ranges of radiobiological parameters, but many others are available. It is also probably the simplest to use. For protons there are some comparative studies on the range of different models (Rorvik et al. 2018; Paganetti et al. 2019; Mara et al, 2020). The study of Mara et al. (2020), showed that the model used in the present study more closely matched the experimental data for cell lines with low $\alpha / \beta$ ratios. The analysis for the higher $\alpha / \beta$ ratio cells by Mara et al. (2020) appears to be affected by the occurrence of low dose hypersensitivity and is presently being revised [Mara, personal communication 2021]. Since the GUI is open source such models could potentially be included in any further extensions, and owners of such models are invited to participate in such further developments by writing to the present authors. It is further proposed that the use of the selected dosefractionation, on retreatment, when using the default proton RBE of 1.1 should be 
compared with that obtained using the LET based RBE model if the relevant tissue LET is known, or can be assumed on the basis of previously published studies. Some clinicians may prefer to adopt an intermediate treatment value of dose per fraction or numbers of fractions between the estimates provided by each of these two approaches.

As in most clinical modelling systems, important caveats are required:

(1) The results are dependent on the validity of the linear quadratic model of radiation effect and on all the assumptions made.

(2) The retreatment dose-fraction estimates represent a boundary condition which should not be exceeded by giving additional dose or BED.

(3) It cannot be over-emphasised that the output estimations act as a guide to patient management and the final dose per fraction, or number of fractions estimated, may be reduced according to the preferences of the treating clinician.

(4) The risks of retreatment must be accepted by the responsible clinician, who should be familiar with the underlying radiobiological principles, and in all cases full informed consent should be obtained after discussions with each individual patient.

(5) Ideally the model should be used as part of a national/international programme which can record outcomes and provide analytical feedback.

(6) The RBE allocations can be modified to include alternative LET-RBE models if necessary by contacting the present authors for such collaboration and to provide the necessary specific codes. 
If used with care, the GUI should allow clinicians and physicists to estimate safe retreatment doses when radiation treatments with photons and protons (or ion beams) are given as separate treatment courses.

\section{Declaration of Competing Interests}

The authors declare no conflict of interest.

\section{Acknowledgements}

JWM acknowledges the support of Cardiff University via funding from the Cardiff Undergraduate Research Opportunities Programme (CUROP) 2019 and Knowledge Economy Skills Scholarships (KESS2), KESS2 is a pan-Wales higher-level skills initiative led by Bangor University on behalf of the Higher Education sector in Wales. It is part-funded by the Welsh Government's European Social Fund (ESF).

\section{Notes on contributors:}

Joshua W. Moore is a PhD candidate in applied mathematics at Cardiff University where he primarily focuses on developing multiscale mathematical models to investigate organoid formation. In addition, his research interests are in applying mathematics in biological areas of sub-cellular dynamics, pattern formation, virology and oncology.

Thomas E. Woolley studied mathematics at the University of Oxford between 2004 and 2017 and is now a Lecturer of Applied Mathematics at Cardiff University. His 
research focuses on applying mathematical techniques to biological problems in pattern formation, stem cell movement, oncology and neurobiology.

John W. Hopewell was the Director of Radiobiological Research, University of Oxford, between 1980 and 2001. His primary research interest has been the study of different aspects of normal tissue toxicity related to radiotherapy: pathogenesis, fractionation and dose-rate, volume effects and retreatment.

Bleddyn Jones is a medically qualified radiobiologist and Professor of Clinical Radiobiology at The University of Oxford. He has made many contributions to explicit mathematical models of radiotherapy and chemotherapy, as well as their application in the clinic. He started re- treating brain and spinal tumour patients in 1991. 


\section{References}

Chapra S. 2012. Applied Numerical Methods with MATLAB for Engineers and Scientists. New York (NY): McGraw- Hill pp. 134-140.

Chen Y, Li J, Li C, Qiu R, Wu Z. 2017. A modified microdosimetric kinetic model for relative biological effectiveness calculation. Phys. Med. Biol. 63: 015008

Elsässer T, Krämer M, Scholz M, 2008. Accuracy of the local effect model for the prediction of biologic effects of carbon ion beams in vitro and in vivo. Int. J. Radiat. Oncol. Biol. Phys. 71: 866-872.

Friedrich T, Scholz U, Elsässer T, Durante M, Scholz M. 2013. Systematic analysis of RBE and related quantities using a database of cell survival experiments with ion beam irradiation. J. Radiat. Res. 54: 494-514.

Grassberger C, Trofimov A, Lomax A, Paganetti H. 2012. Variations in linear energy transfer within clinical proton therapy fields and the potential for biological treatment planning. Int. J. Radiat. Biol. 80: 1559-1566.

Hall EJ, Gaccia A. 2019. In Chapter 20, Clinical Response of Normal Tissue (page 367). Radiobiology for the Radiologist ( $8^{\text {th }}$ edition). Wolters Kluwer, Philadelphia

Jones B, Dale RG, Deehan C, Hopkins KI, Morgan DAL. 2001. The role of biologically effective dose (BED) in clinical oncology. Clin. Oncol. 3: 71-81.

Jones B, Dale RG, Gaya A. 2006. Linear quadratic modelling of increased late normal tissue effects in special clinical situations. Int. J. Radiat. Oncol. Biol. Phys. 64: 948953.

Jones B, Grant W. 2014. Retreatment of Central Nervous System Tumours Statement of Search Strategies Used and Sources of Information. Clin. Oncol. 26: 407-18.

Jones B, Hill MA. 2019. Physical characteristics at the turnover-points of relative biological effect (RBE) with linear energy transfer (LET). Phys. Med. Biol. 64: 22501064.

Jones B, Hopewell JW. 2014. Alternative models for estimating the radiotherapy retreatment dose for the spinal cord. Int. J. Radiat. Biol. 90: 731-41.

Jones B, Hopewell JW. 2019. Spinal cord re-treatments using photon and proton based radiotherapy: LQ-derived tolerance doses. Phys. Medica 64: 304-310. 
Jones B. 2016. Why RBE must be a variable and not a constant in proton therapy. Br. J. Radiol. 89: 20160116.

Jones B. 2017. Clinical radiobiology of proton therapy: modelling of RBE. Acta Oncol. 56: $1374-1378$.

Jones B. 2015(a). Towards achieving the full clinical potential of proton therapy by inclusion of LET and RBE models. Cancers 7: 460-480.

Jones B 2015(b). A simpler energy transfer efficiency model to predict relative biological effect (RBE) for protons and heavier ions. Front. Oncol.5: 184. (Erratum: Front. Oncol. 2016; 6: 32).

Lu hr A, von Neubeck C, Helmbrecht S, Baumann M, Enghardt W, Krause M. 2017. Modeling in vivo relative biological effectiveness in particle therapy for clinically relevant endpoints. Acta Oncol. 56: 1392-1398.

Mara E, Clausen M, Khachonkham S, Deycmar S, Pessy C, Dörr W, Kuess P, Georg $D$, Gruber $S$. 2020. Investigation of the impact of $\alpha / \beta$ and LET on relative biological effectiveness in scanned proton beams: an in vivo study based on human cell lines. Med. Phys. 47: 3691-3702.

Muirhead R, Jones B. 2018. Re-irradiation is now a real option - but how do we take it forward? Clin. Oncol, (Royal Coll Radiologists), 30: 65-66.

Paganetti H, Blakely E, Carabe-Fernandez A et al. 2019. Report of the AAPM TG-256 on the relative biological effectiveness of proton beams in radiation therapy. Med. Phys. 46: e53-e78.

Rorvik E, Fjera LF, Dahle TJ, Dale JE, Engeseth GM, Stokkevag CH, Tho rnquist S, Ytre- Hauge KS. 2018. Exploration and application of phenomenological RBE models for proton therapy. Phys. Med. Biol. 63: 185013.

Ruifrok AC, Stephens LC, van der Kogel AJ. 1994. Radiation response of the rat cervical spinal cord after irradiation at different ages: tolerance, latency and pathology. Int. J. Radiat. Oncol. Biol. Phys. 29: 73-99.

Saager M, Peschke P, Brons S, Debus J, Karger CP. 2018. Determination of the proton RBE in the rat spinal cord: Is there an increase towards the end of the spread-out Bragg peak? Radiother. Oncol. 128: 115-120.

T E Schultheiss, Kun LE, Ang KK, Stephens LC. 1995. Radiation response of the central nervous system. Int. J. Radiat. Oncol. Biol. Phys. 31: 1093-1112. 
Woolley TE, Belmonte-Beitia J, Calvo GF, Hopewell JW, Gaffney EA, Jones B. 2018. Changes in the retreatment radiation tolerance of the spinal cord with time after the initial treatment. Int. J. Radiat. Biol. 94: 515-531.

van der Kogel AJ. 1989. Editorial. Continuous hyperfractionated accelerated radiotherapy (CHART). Radioth. Oncol.: 16: 75-77. 


\section{Appendix 1}

The revised Graphical User Interface (GUI) described in this manuscript can be downloaded as zip file from the Supplementary data associated with this manuscript or from an online repository at http://bit.ly/BED_CAL. This contains a Java .exe file and the associated font file. A ReadMe file sets the terms for use of the GUI along with guidelines (SUPPLEMENTARY MATERIAL 1 and 2).

\section{Appendix 2}

The present study aims to encourage the use of mathematical modelling in the planning of CNS retreatments using photon, proton and carbon ion therapy modalities. The development of the universally accessible software integrates existing data-driven retreatment dose estimation models to allow for swift results without the need for manual calculations. A fundamental concept of retreatment dose estimation relies on tissue recovery over elapsed time following a linear-quadratic relationship for the maximum dose required to achieve a specific biological effect (Jones and Hopewell 2014). This relationship is denoted as the biological effective dose (BED), and is specified for the number of treatment fractions, $\mathrm{n}$, the fractional dose, $\mathrm{d}$, by the following equation

$$
B E D=n d\left(1+\frac{d}{\alpha / \beta}\right)
$$

where $\alpha / \beta$ represents tissue sensitivity to the total radiation dose $n d$. BED estimation was initially developed for photon therapies, however, there has been recent efforts to demonstrate a photon-proton (ion) BED equivalence via the inter-relationship between linear energy transfer (LET) and relative biological effectiveness (RBE) (Jones and Hopewell 2019). Therefore, a (i) time-dependent tissue recovery photon BED model (Woolley et al. 2018) has been coupled to a (ii) photon-proton (ion) BED equivalence 
model (Jones and Hopewell, 2019) to produce a highly flexible retreatment dose estimation calculator for any combination of photon and proton (ion) initial treatment and retreatment.

The time-dependent tissue recovery photon BED model developed by Woolley and colleagues (2018) extends previous retreatment dose estimation BED models (Jones and Hopewell 2014; Jones and Grant 2014) to consider treatment intervals within a year (Woolley et al. 2018). The retreatment BED $\left(\mathrm{BED}_{\text {ret }}\right)$ is determined via a nondimensional scaling $\left(\mathrm{BED}_{2}\right)$ with the maximal tolerance $\mathrm{BED}\left(\mathrm{BED}_{\mathrm{R} \%}\right)$ that is dependent on the associated risk of myelopathy. There exists a similar non-dimensional $\left(\mathrm{BED}_{1}\right)$ scaling for the initial treatment $\mathrm{BED}\left(\mathrm{BED}_{\text {init }}\right)$ and $\mathrm{BED}_{\mathrm{R} \%}$ (using the same tolerance) that is fully determined via clinical input data. These relations can be described explicitly as

$$
\begin{aligned}
& B E D_{\text {init }}=\frac{B E D_{1} \cdot B E D_{R \%}}{100}, \#(2) \\
& B E D_{\text {ret }}=\frac{B E D_{2} \cdot B E D_{R \%}}{100} . \#(3)
\end{aligned}
$$

Therefore, the aim for retreatment dose estimation is to define a relationship between $\mathrm{BED}_{1}$ and $\mathrm{BED}_{2}$ which allows for tissue recovery between treatments. Namely, equations (2) and (3) are coupled by the following,

$$
B E D_{2}=100\left(1-\frac{B E D_{1}}{100}\right)\left(1+\left(\left(1-\frac{B E D_{1}}{100}\right)^{\frac{-r(t)}{1+r(t)}}-1\right) f\left(B E D_{1}, r(t)\right)\right), \#(4)
$$

where $r(t)$ is a time-dependent the tissue recovery function that can be approximated by a cubic polynomial to fit existing data (Jones and Grant 2014; Woolley et al. 2018). The inclusion of the function $f\left(B E D_{1}, r(t)\right)$ in equation (4) was imposed to yield an 
adaptive measure to control the transition between initial BED-dependent early and later recovery. Specifically, $f\left(B E D_{1}, r(t)\right)$ is bounded between 0 and 1 and has the form,

$$
f\left(B E D_{1}, r(t)\right)=\frac{1}{2}\left(1+\tanh \left(s_{0}\left(B E D_{1}-\frac{\underline{B E D}}{1+s_{1} r(t)}\right)\right)\right), \quad \#(5)
$$

such that parameters $s_{0}, s_{1}$ and $\underline{B E D}$ control the aforementioned transition from early to late tissue recovery, that is, $f\left(B E D_{1}, r(t)\right) \approx 0$ corresponds to no recovery and $f\left(B E D_{1}, r(t)\right) \approx 1$ represents the recovery model of Jones and Hopewell (2014). For a detailed justification of the functional forms for BED retreatment estimation (equations (\#4-5)) and parameter estimation, see Woolley et al. (2018).

To include proton and carbon ions into the tissue recovery retreatment BED estimation, the BED equivalence model developed by Jones and Hopewell (2019) was introduced into the model. The association between a photon therapy and proton (ion) therapy is dependent on RBE of proton therapy, such that the photon equivalent dose $\left(d_{e q}\right)$ for a proton treatment of dose $d_{p}$ in $n$ fractions is given by,

$$
d_{e q}=d_{p} \cdot R B E, \#(6)
$$

and therefore, can be directly substituted into equation (\#1) for use in the photon tissue recovery model (equations (\#2-3)). The value RBE is dependent on the LET the of ionising particles and the proton physical dose $d_{p}$. In particular, the RBE can be determined from the ratio of a dose with respect to a lower reference-radiation sensitivity tolerance and the proton physical dose, that is,

$$
R B E=\frac{d_{\text {low }}}{d_{p}}
$$

The lower reference dose can be estimated from the operative LET value $\left(\mathrm{LET}_{\mathrm{x}}\right)$, control LET value $\left(\mathrm{LET}_{\mathrm{c}}\right)$ and maximum bio-efficiency LET value $\left(\mathrm{LET}_{\mathrm{u}}\right)$, from the following equation 


$$
d_{\text {low }}=\frac{-\alpha_{\text {low }}+\sqrt{\alpha_{\text {low }}^{2}+4 \beta_{\text {low }} d_{p}\left(\alpha_{h i}+\beta_{\text {hi }} d_{p}\right)}}{2 \beta_{\text {low }}}, \# \text { (8) }
$$

where $\alpha_{\text {low }}$ and $\beta_{\text {low }}$ are the reference-radiation $\alpha$ and $\beta$ tissue sensitivity values and $\alpha_{h i}$ and $\beta_{h i}$ are calculated via,

$$
\begin{gathered}
\alpha_{h i}=\alpha_{\text {low }}+\frac{\left(2.7\left(1-e^{-3.9 \alpha_{\text {low }}}\right)-\alpha_{\text {low }}\right)\left(L E T_{x}-L E T_{c}\right)}{\left(L E T_{u}-L E T_{c}\right)}, \#(9) \\
\beta_{\text {hi }}=\beta_{\text {low }}+\frac{\left(0 . 0 6 \left(1-e^{\left.\left.-50 \beta_{\text {low }}\right)-\beta_{\text {low }}\right)\left(L E T_{x}-L E T_{c}\right)}\right.\right.}{\left(L E T_{u}-L E T_{c}\right)}, \#(10)
\end{gathered}
$$

which are defined empirically. This analytic association between proton and photonbased therapies allows for acute retreatment dose estimations for any combination of proton/photon treatment and retreatment when coupled to the photon-based tissue recovery model (equations (\#2-3)).

The software accompanying this study produces combines the previously discussed models as demonstrated in computational flow diagram in Figure A1. The initial treatment is inputted as the total dose and number of dose fractions. Proton (ion) doses are converted to equivalent photon dose using an RBE factor, so that the initial treatment can be assigned a $B E D_{\text {init }}$ for the level of risk considered acceptable on retreatment, including conservatism thought appropriate on clinical grounds. The $B E D_{\text {ret }}$ is then converted back to an acceptable photon treatment directly or via an $\mathrm{RBE}$ factor if retreatment is to be with protons (ions). 


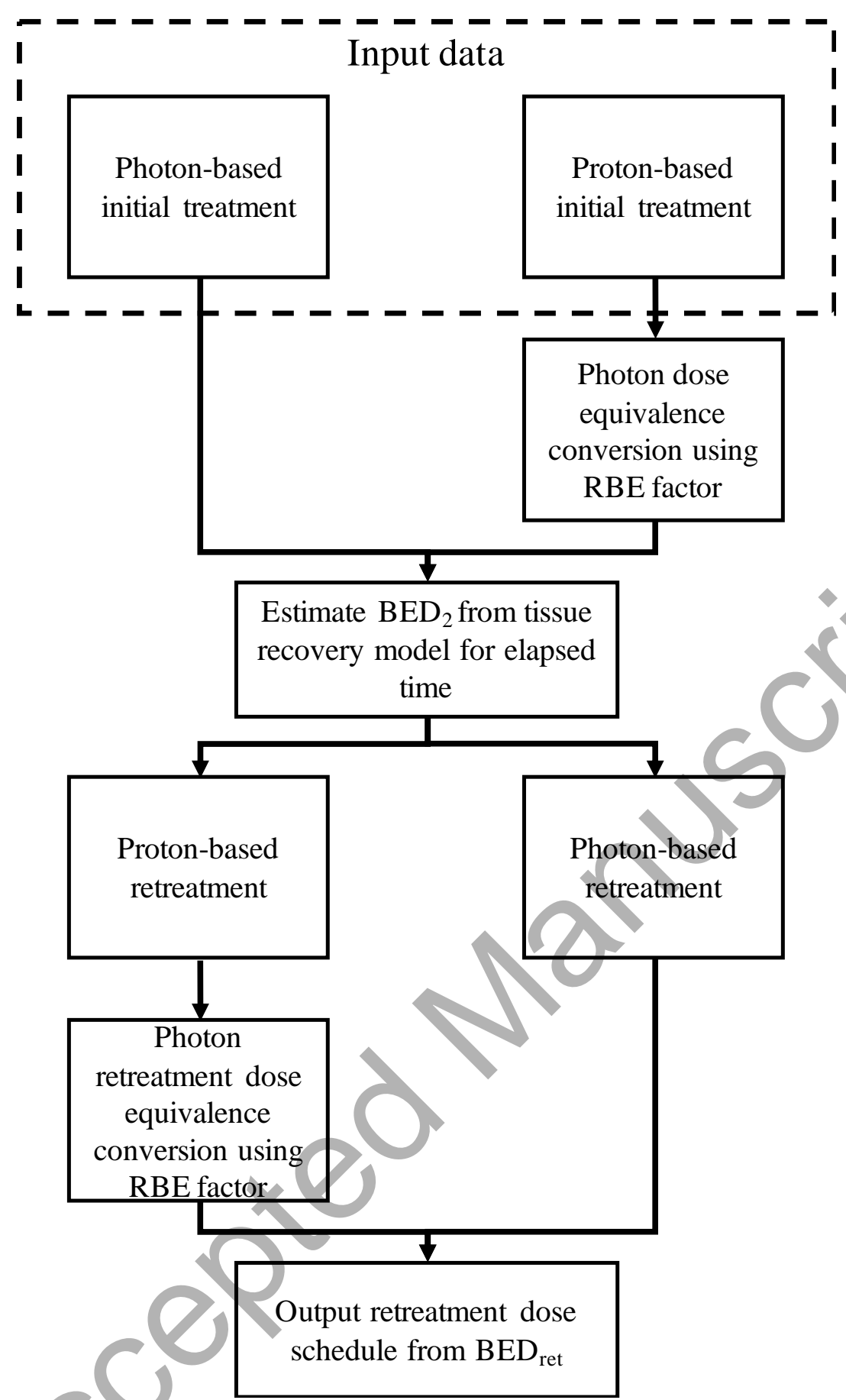

Figure A1. Computational pipeline for the BED calculation for both Photon and Proton treatments. 


\section{Text to Figures}

Figure 1. Illustrates the use of the BED calculator for worked example 1, for a PhotonPhoton treatment plan. All parameters associated with the time-dependent radiotherapy model ( $\mathrm{s}_{0}, \mathrm{~s} 1$ and $\left.\underline{\mathrm{BED}}\right)$ were defined previously (Woolley et al. 2018).

Figure 2(a). Photon-Proton treatment with variable, LET derived, RBE. Figure 2(b). Photon-Proton treatment using the default fixed RBE of 1.1.

Figure 2. Illustrates of the use of the BED calculator for worked example 2, for a Photon-Proton treatment plan using either a variable (a) or a fixed (b) RBE value. All parameters associated with the time-dependent radiotherapy model $\left(\mathrm{s}_{0}, \mathrm{~s} 1\right.$ and $\left.\underline{\mathrm{BED}}\right)$ were defined previously (Woolley et al. 2018).

Figure 3. Illustrates the use of the BED calculator for worked example 3, for a ProtonPhoton treatment plan. All parameters associated with the time-dependent radiotherapy model ( $\mathrm{s}_{0}, \mathrm{~s} 1$ and $\left.\underline{\mathrm{BED}}\right)$ were defined previously (Woolley et al. 2018).

Figure 4. Illustrates the use of the BED calculator for worked example 4, for a ProtonProton treatment plan, using a LET derived RBE for protons. All parameters associated with the time-dependent radiotherapy model ( $\mathrm{s}_{0}, \mathrm{~s} 1$ and $\left.\underline{\mathrm{BED}}\right)$ were defined previously (Woolley et al. 2018).

Figure 5. Illustrates the use of the BED calculator for worked example 5, for a carbon ion - carbon ion treatment plan. All parameters associated with the time-dependent radiotherapy model ( $\mathrm{s}_{0}, \mathrm{~s} 1$ and $\underline{\mathrm{BED}}$ ) were defined previously (Woolley et al. 2018).

Figure A1. Computational pipeline for the BED calculation for both Photon and Proton treatments. 


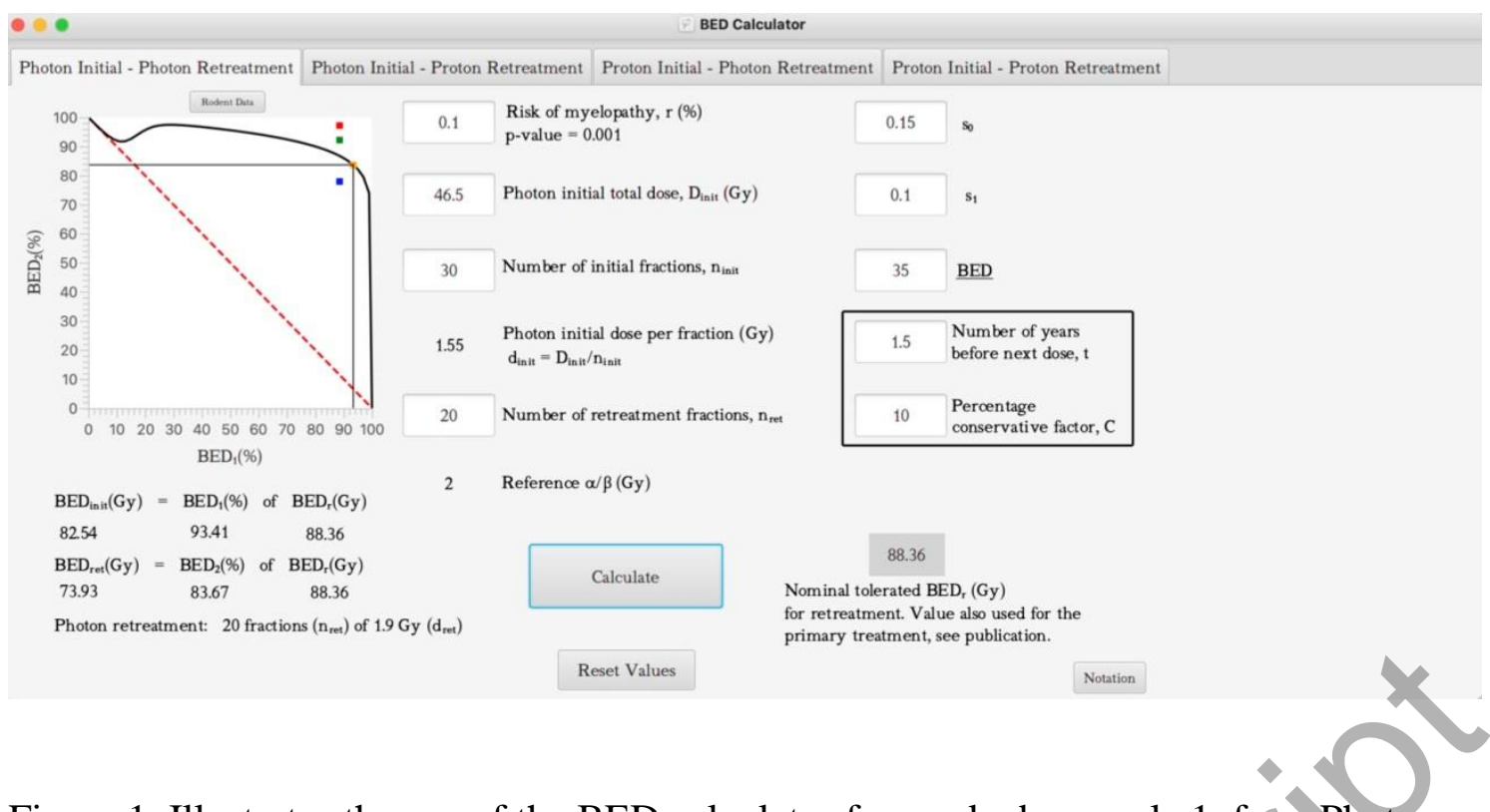

Figure 1. Illustrates the use of the BED calculator for worked example 1, for a PhotonPhoton treatment plan. All parameters associated with the time-dependent radiotherapy model (s0, s1 and BED) were defined previously (Woolley et al. 2018). 


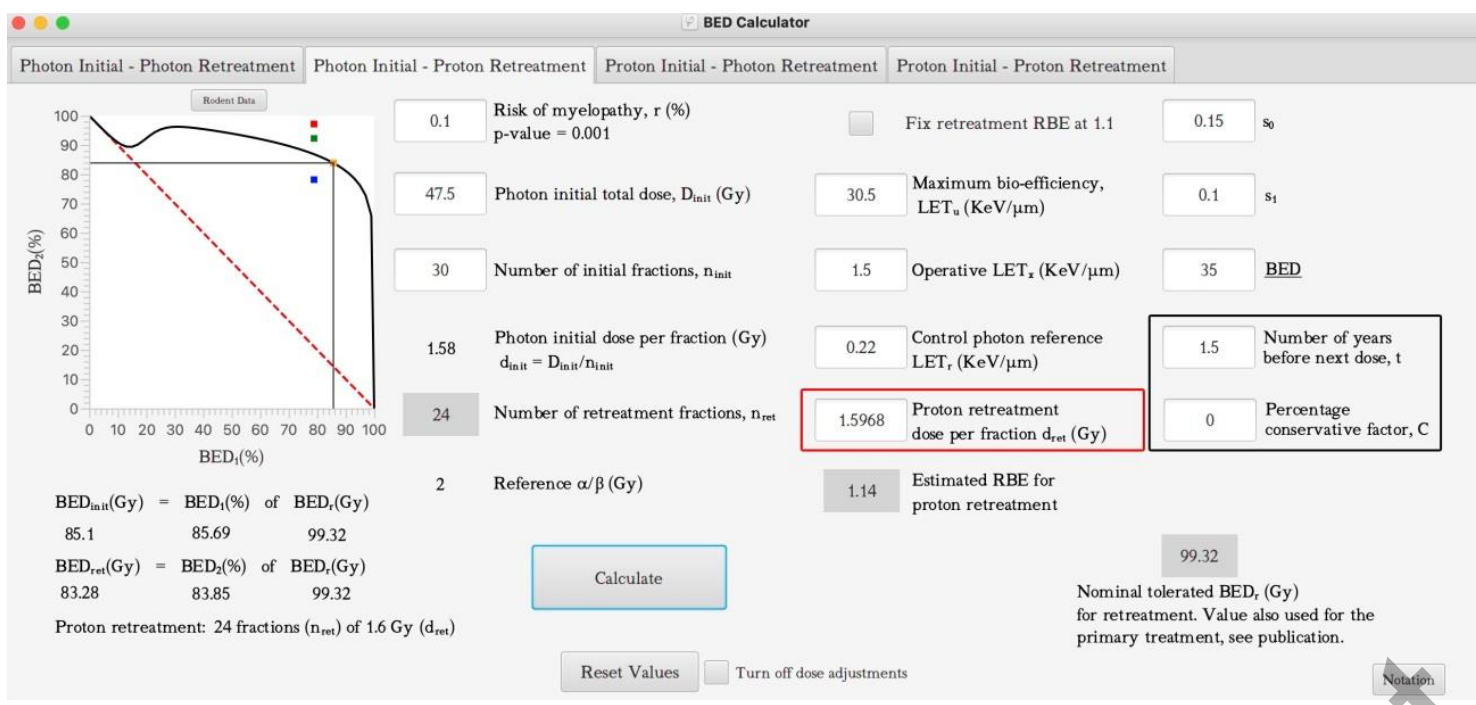

(a) Photon-Proton treatment with variable, LET derived, RBE.

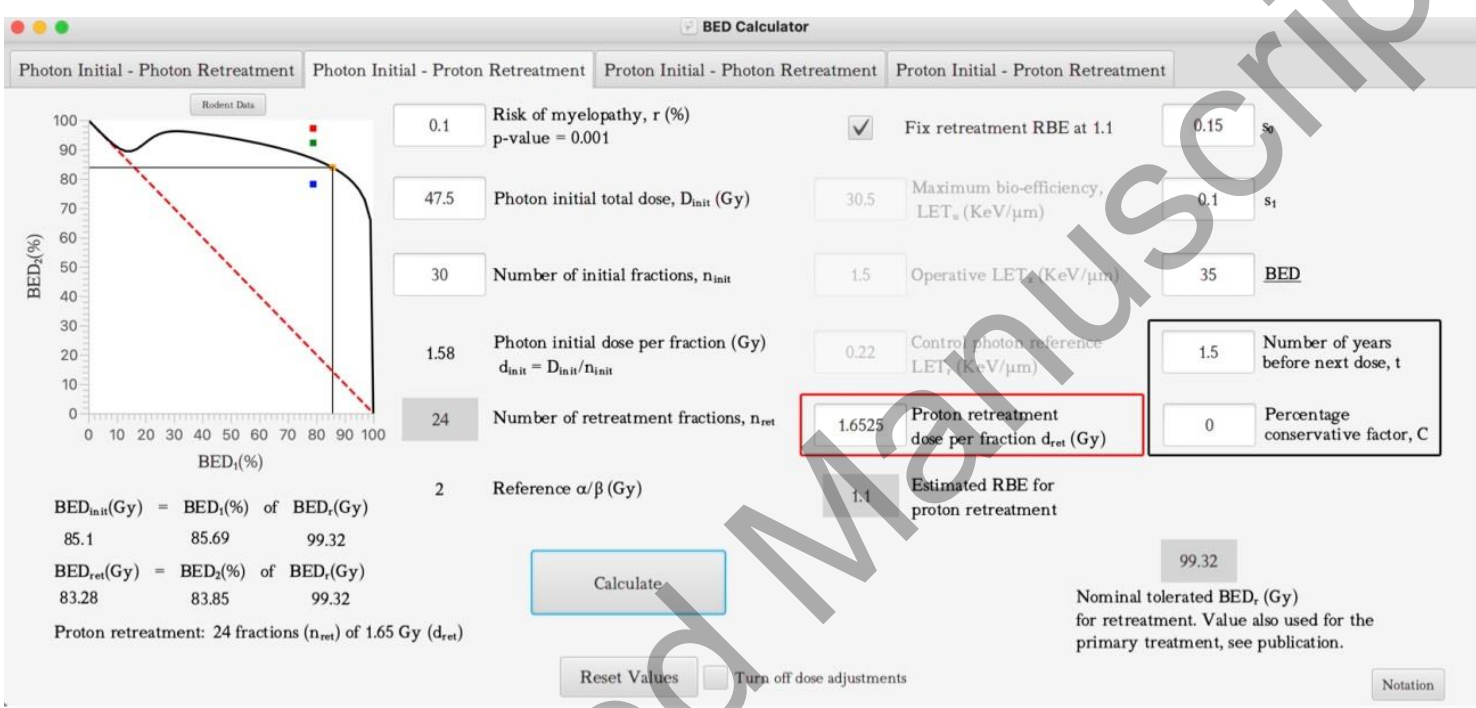

(b) Photon-Proton treatment using the default fixed RBE of 1.1.

Figure 2. Illustrates the use of the BED calculator for worked example 2, for a Photon-

Proton treatment plan using either a variable (a) or a fixed (b) RBE value. All

parameters associated with the time-dependent radiotherapy model (s0, s1 and BED)

were defined previously (Woolley et al. 2018). 


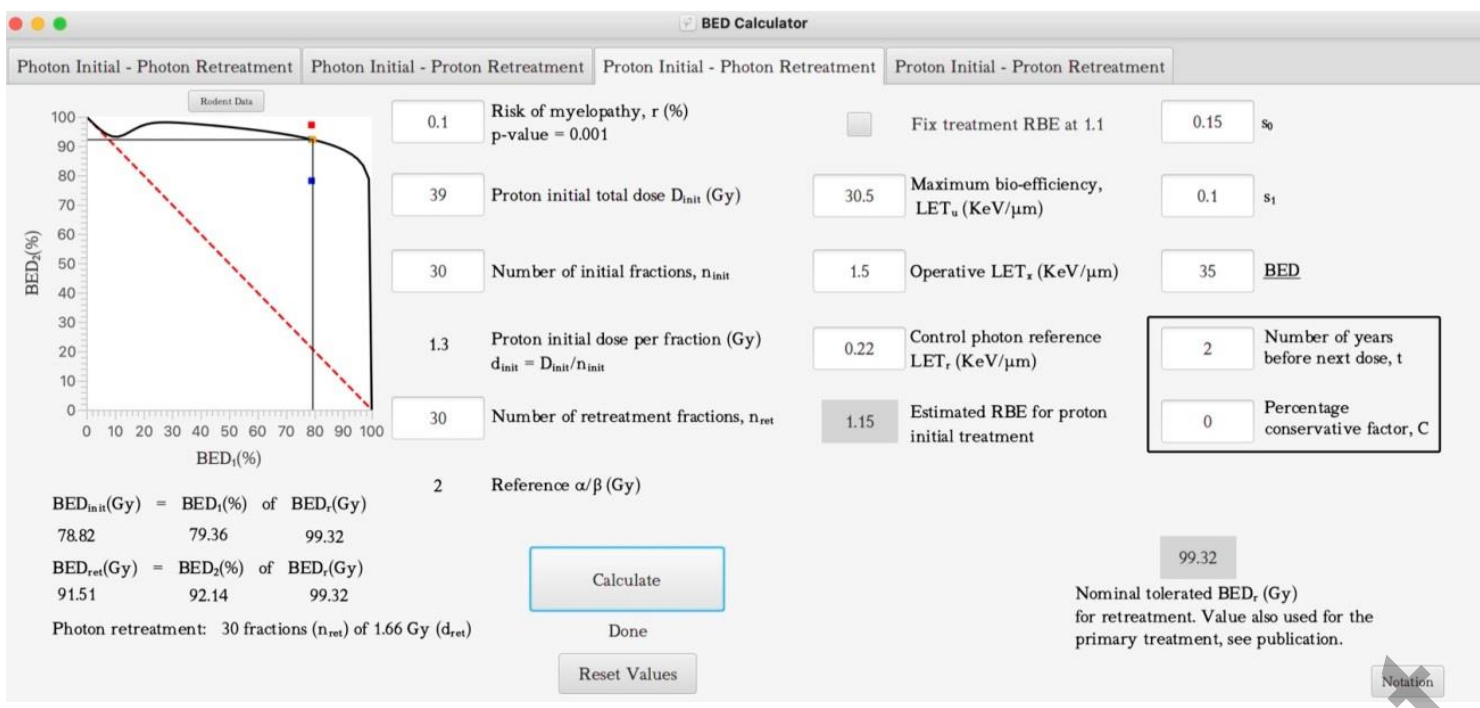

Figure 3. Illustrates the use of the BED calculator via worked example 3, for a ProtonPhoton treatment plan. All parameters associated with the time-dependent radiotherapy model (s0, s1 and BED) were defined previously (Woolley et al. 2018). 


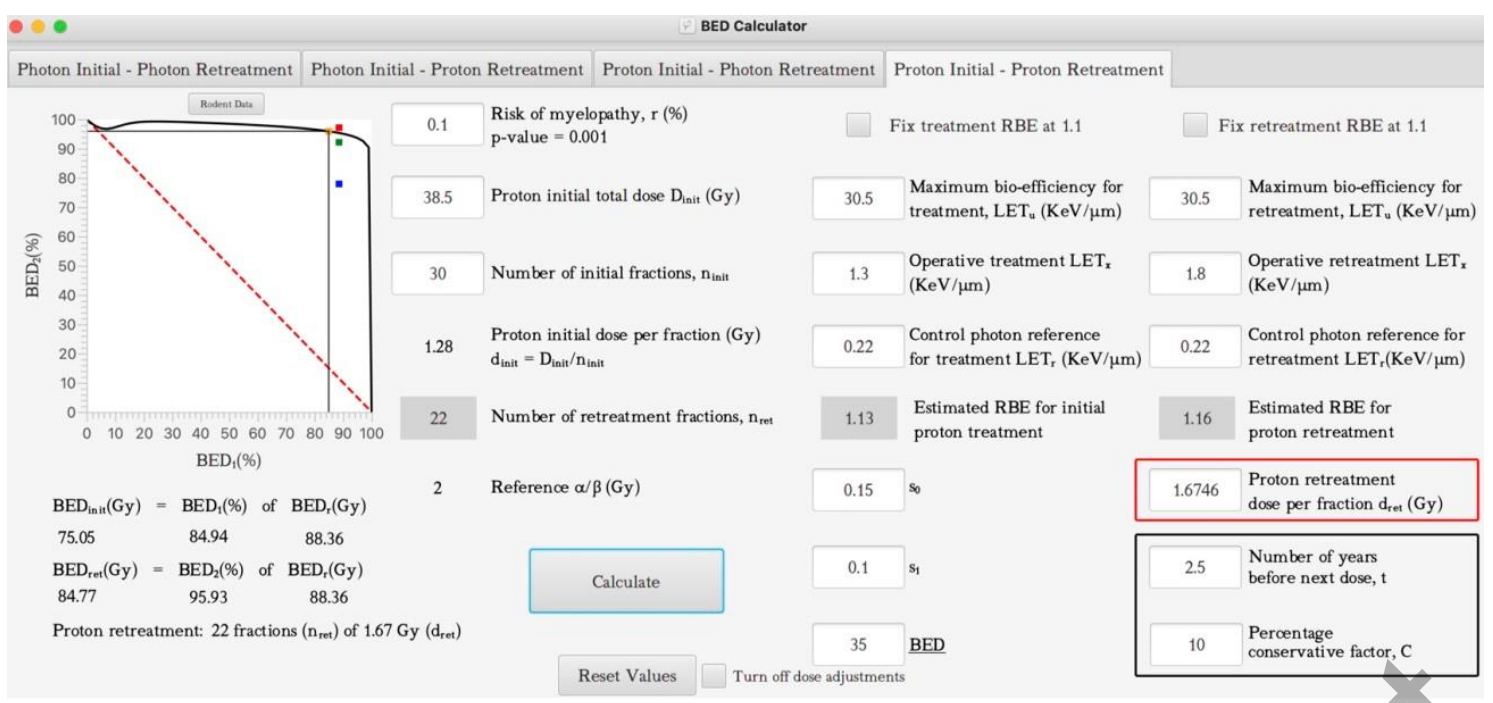

Figure 4. Illustrates the use of the BED calculator for worked example 4, for a Proton-

Proton treatment plan, using a LET derived RBE for protons. All parameters associated with the time-dependent radiotherapy model ( $\mathrm{s} 0, \mathrm{~s} 1$ and BED) were defined previously (Woolley et al. 2018). 


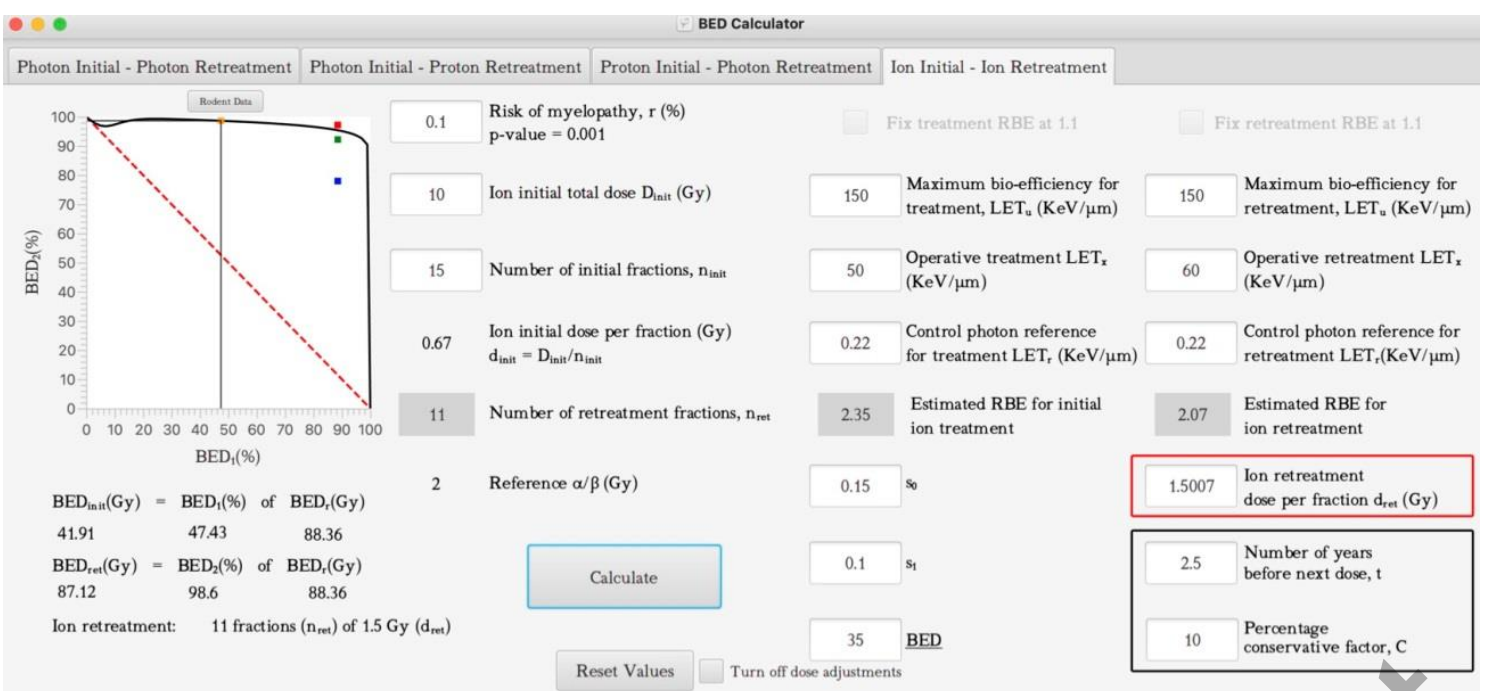

Figure 5. Illustrates the use of the BED calculator for worked example 5, for a carbon ion - carbon ion treatment plan. All parameters associated with the time-dependent radiotherapy model (s0, s1 and BED) were defined previously (Woolley et al. 2018). 